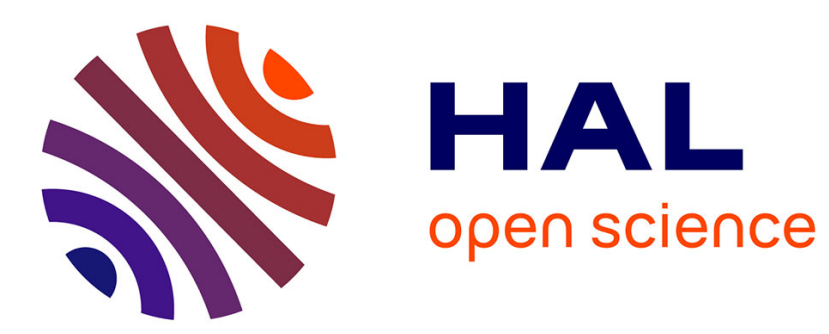

\title{
Filtered Tabulated Chemistry for non-premixed flames
} Axel Coussement, Schmitt Thomas, Benoit Fiorina

\section{To cite this version:}

Axel Coussement, Schmitt Thomas, Benoit Fiorina. Filtered Tabulated Chemistry for non-premixed flames. Proceedings of the Combustion Institute, 2015, 35 (2), 10.1016/j.proci.2014.06.010 . hal01347930

\section{HAL Id: hal-01347930 \\ https://hal.science/hal-01347930}

Submitted on 30 Mar 2020

HAL is a multi-disciplinary open access archive for the deposit and dissemination of scientific research documents, whether they are published or not. The documents may come from teaching and research institutions in France or abroad, or from public or private research centers.
L'archive ouverte pluridisciplinaire HAL, est destinée au dépôt et à la diffusion de documents scientifiques de niveau recherche, publiés ou non, émanant des établissements d'enseignement et de recherche français ou étrangers, des laboratoires publics ou privés. 


\title{
Filtered Tabulated Chemistry for non-premixed flames
}

\author{
A. Coussement, T. Schmitt*, B. Fiorina \\ EM2C Laboratory, CNRS, Ecole Centrale Paris, Grande Voie des Vignes, 92295 Chatenay-Malabry, France
}

The objective of the present study is to design a modeling strategy for LES of laminar diffusion flame regimes, i.e. without SGS wrinkling. A non-premixed model dedicated to capture unresolved laminar flame structure is then proposed. For that purpose, the Filtered Tabulated Chemistry for Large Eddy Simulation is adapted to diffusion flames. A filtered look-up table computed from a collection of strained 1-D counterflow flames is generated. The filtered flame structure and thickness is captured with three controlling variables which are the filtered mixture fraction, the filtered progress variable and the filter size. This approach is successfully applied to 1-D and 2-D unresolved counter-flow flame simulations. The filter size governs the minimal thickness of the filtered thermal layer.

\section{Introduction}

Numerous non-premixed combustion LES models rely on a statistical description of flame turbulence interactions [1]. Commonly used approaches to estimate subgrid Filtered Density Function (FDF) are based on probability transport equations [2], Condition Moment Closure $[3,4]$ or presumed FDF $[5,6]$. These approaches are performing well in the presence of high level of turbulent wrinkling but do not guarantee that the proper flame structure is recovered when the wrinkling is fully resolved or in laminar cases.

This situation is for instance encountered in the simulation of liquid rocket engines, that operates at very high pressures, typically around $10 \mathrm{MPa}$. Indeed, experimental investigations have shown that the coaxial injection system promotes the development of a turbulent non-premixed jet flame anchored at the injector lips [7,8]. Direct numerical simulation of this near-burner region show that the flame front is very thin (of the order of $10-100 \mu \mathrm{m}$ ) and weakly wrinkled by turbulent motions $[9,10]$. Because of numerical resolution issues, Large Eddy Simulation of such flame regime is extremely challenging. This is illustrated in Fig. 1, which shows a schematic view of a developing turbulent reactive mixing layer behind a splitter plate. At the flame basis, the flame thickness $\delta_{Z}^{0}=1 / \max |\nabla Z|$, where $Z$ is the mixture fraction, is much thinner than the LES grid size used in practical configuration.

The objective of the present study is to discuss modeling issues in LES of laminar diffusion flame regimes, i.e. when no flame wrinkling occurs at the Sub Grid Scale (SGS). A modeling strategy, which controls the filtered flame thickness on unresolved situations i.e. when the flame thickness is smaller 


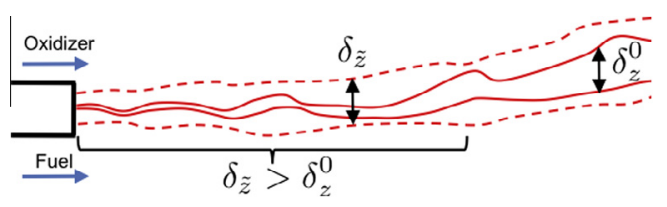

Fig. 1. Schematic representation of a diffusion flame stabilization behind a splitter plate. The continuous line represents the unfiltered flame brush, while the dashed line shows the filtered flame that is required to be properly handled by a LES mesh. $\delta_{z}^{0}$ is the flame thickness and $\delta_{\tilde{z}}$ is the filtered flame thickness.

than the grid size, is proposed. In Section 2, the structure of a filtered non-premixed flame front is analyzed by post-processing a collection of 1-D filtered counterflow detailed chemistry flames. In Section 3, a combustion model is developed in the framework of the Filtered Tabulated Chemistry model for LES (F-TACLES), initially developed for premixed [11] and stratified [12] combustion. In Section 4, the model is implemented in a 1-D Low Mach number counter-flow solver and in a multi-dimensional compressible flow solver. Numerical simulations of filtered (unresolved) diffusion flames are presented.

\section{The chemical structure of a filtered laminar diffusion flame}

\subsection{Conservation equations and numerical configuration}

The chemical structure of non-premixed combustion is investigated on 1-D counterflow flame configurations, governed by the following set of balance equations [13]:

$$
\begin{aligned}
& \frac{\partial \rho}{\partial t}+\frac{\partial \rho u_{n}}{\partial x_{n}}+2 \epsilon \rho U=0 \\
& \rho \frac{\partial U}{\partial t}+\rho u_{n} \frac{\partial U}{\partial x_{n}}-\epsilon\left(\rho U^{2}-\rho_{\infty}\right)=\frac{\partial}{\partial x_{n}}\left(\mu \frac{\partial U}{\partial x_{n}}\right) \\
& \rho \frac{\partial Y_{k}}{\partial t}+\rho u_{n} \frac{\partial Y_{k}}{\partial x_{n}}=-\frac{\partial \rho V_{k} Y_{k}}{\partial x_{n}}+\dot{\omega}_{k} \\
& \rho c_{p} \frac{\partial T}{\partial t}+\rho c_{p} u_{n} \frac{\partial T}{\partial x_{n}}=\frac{\partial}{\partial x_{n}}\left(\lambda \frac{\partial T}{\partial x_{n}}\right) \\
& \quad-\left(\rho \sum_{k=1}^{k=N_{s p}} c_{p, k} Y_{k} V_{k}\right) \frac{\partial T}{\partial x_{n}}-\sum_{k=1}^{k=N_{s p}} h_{k} \dot{\omega}_{k}
\end{aligned}
$$

where $x_{t}$ and $x_{n}$ are the spatial coordinates in the direction parallel and normal to the flame front, respectively. $U\left(x_{n}\right)=u_{t} / \epsilon x_{t}$, where $\epsilon$ is the strain rate and $u_{n}$ and $u_{t}$ are the velocities normal and parallel to the flame front, respectively. $Y_{k}$ and $\dot{\omega}_{k}$ are the species mass fraction and chemical mass reaction rate of kth species, respectively. $T$ is the temperature, $\rho$ is the density, $c_{p}$ is the mixture heat capacity, $h_{k}$ is the enthalpy per unit of mass of species $k$ and $V_{k} Y_{k}=-D_{k} \partial Y_{k} / \partial x_{n}$ with $D_{k}$ the diffusion coefficient of species $k$. Thermochemical quantities are computed using GRI 3.0 detailed chemistry scheme [14] which involves 53 species and 325 elementary reactions.

To highlight the influence of filtering on diffusion flames, pure methane and pure oxygen $\left(p=101325 \mathrm{~Pa}, \quad T_{i n j, \mathrm{O}_{2}}=T_{i n j, \mathrm{CH}_{4}}=300 \mathrm{~K}\right)$ onedimensional counterflow diffusion flames are computed for various strain rates $\epsilon$. Calculations are done under unity Lewis number assumption.

\subsection{Filtered counter-flow diffusion flames}

Figure 2 plots the mixture fraction $Z$ (equal to 0 and 1 in the oxidizer and fuel streams, respectively) and temperature $T$ of the counterflow $\mathrm{CH}_{4} / \mathrm{O}_{2}$ flame configuration as a function of $x_{n}$ for two different strain rates: $\epsilon=1000 \mathrm{~s}^{-1}$ and $\epsilon=10000 \mathrm{~s}^{-1}$. The corresponding resolved flame thicknesses $\delta_{Z}=1 / \max (|\nabla Z|)$ are $1.09 \mathrm{~mm}$ and $0.34 \mathrm{~mm}$, respectively.

To mimic LES conditions, mixture fraction and flow thermo-chemical variables solutions are spatially filtered using a gaussian filter operator of width $\Delta=1 \mathrm{~mm}$. Filtered flame solutions are superimposed in Fig. 2. Resulting filtered flame thicknesses $\delta_{\bar{Z}}=1 / \max (|\nabla \bar{Z}|)$ equal $1.47 \mathrm{~mm}$ for $\epsilon=1000 \mathrm{~s}^{-1}$ and $0.86 \mathrm{~mm}$ for $10000 \mathrm{~s}^{-1}$.
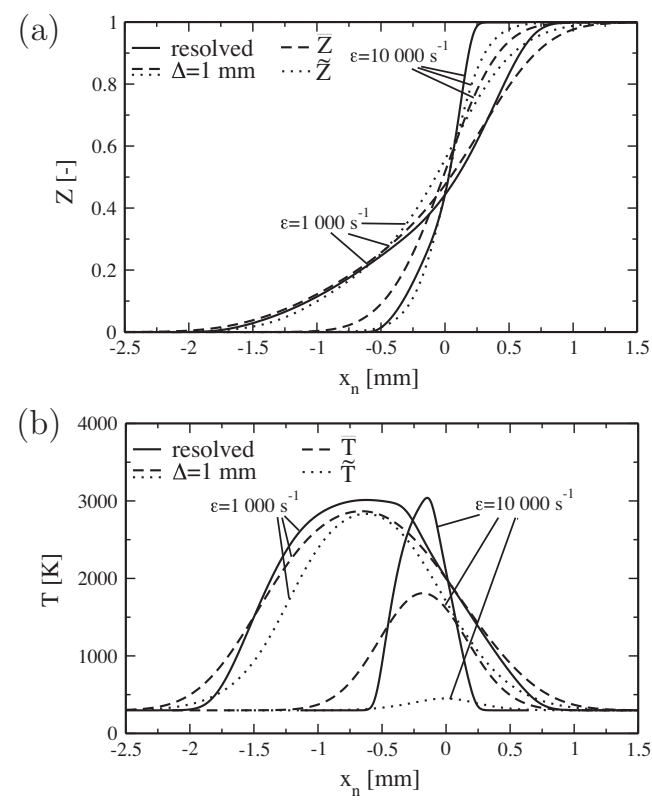

Fig. 2. Impact of filtering on the flame structure (a) Mixture fraction $Z$ and (b) temperature $T$ of a counter flow diffusion flame for strain rates $\epsilon=1000 \mathrm{~s}^{-1}$ and $10000 \mathrm{~s}^{-1}$. Resolved flames and filtered flames with filter size $\Delta=1 \mathrm{~mm}$ are shown. 


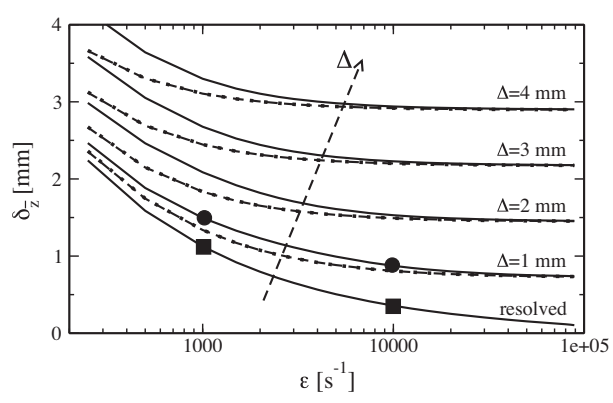

Fig. 3. Flame thickness $\delta_{z}$ as a function of the strain rate $\epsilon$ for resolved flames and a set of filtered flames with $\Delta$ ranging from 1 to $4 \mathrm{~mm}$. and $\bullet$ correspond to cases plotted in Fig. 2. - a priori filtered - - using Eq. (21) ... using Eq. (21) and assuming fast chemistry.

\subsection{Influence of filtering on the flame thickness}

For $\epsilon=1000 \mathrm{~s}^{-1}, \delta_{z} \approx \Delta$. Consequently, as shown in Fig. 2(a), the flame thickness is weakly modified by the filtering operation. But when the strain increases to $10000 \mathrm{~s}^{-1}, \delta_{z}<\Delta$, causing an increase of $\delta_{\bar{Z}} / \delta_{Z}$. The filtering operator then broaden the mixture fraction profile.

The filtered flame thickness has been computed for a large range of strain rates (up to $10^{5} \mathrm{~s}^{-1}$ ) and filter sizes (up to $4 \mathrm{~mm}$ ) to mimic practical LES situations. $\delta_{\bar{Z}}$ is plotted as a function of the strain rate in Fig. 3. For small filter sizes, the condition $\Delta<\delta_{Z}$ is verified and $\delta_{\bar{Z}} \approx \delta_{Z}$. Because $\delta_{Z}$ decreases with the strain rate, the filtered flame thickness first decreases as well. But, above a given strain rate, $\delta_{Z}$ becomes smaller than the filter size, and it is observed that the filtered flame thickness tends to a limit, here around $0.72 \Delta$.

The influence of filter size and strain rate on the filtered flame thickness is qualitatively retrieved by a simple analytical analysis, detailed in Appendix A. Two analytical solutions, which consider or not finite rate chemistry to model

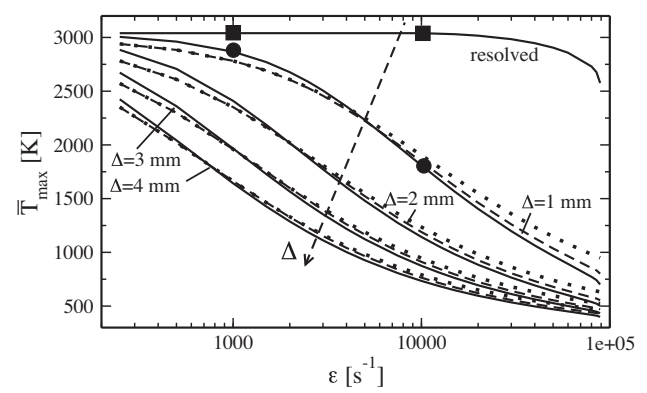

Fig. 4. Maximum flame temperature $T_{\max }$ as a function of the strain rate $\epsilon$ for resolved flames and a set of filtered flames with $\Delta$ ranging from 1 to $4 \mathrm{~mm}$. and $\bullet$ correspond to cases plotted in Fig. 2. - a priori filtered -- using Eq. (20) $\cdots$ using Eq. (20) and assuming fast chemistry. the influence of strain rate, are added to Fig. 4. It is interesting to note that the dependency of the filtered flame thickness to the strain rate is well recovered. The influence of detailed chemistry effects is limited, as the infinitely fast chemistry assumption remains valid, especially for high filter sizes values. Departures, observed at low strain rates, are mainly due to the analytical presumed form of the resolved mixture fraction profile.

\subsection{Influence of filtering on the temperature profile}

As shown on the temperature field plotted in Fig. 2(b), filtering has also a strong impact on the flame structure. Again, the filtering becomes especially effective for high strain rate value (here for $\epsilon=10000 \mathrm{~s}^{-1}$ ), when $\delta_{z}<\Delta$. It causes a decrease of maximum temperature and the broadening of the profile. Favre filtered quantities defined as $\bar{\rho} \tilde{\Phi}=\overline{\rho \Phi}$ are also added to Fig. 2 . Because of the large density variation across the flame front, Favre filtered $(\tilde{T})$ temperature profiles strongly departs from the Reynolds filtered ones $(\bar{T})$. The maximum flame temperature $\bar{T}$ is plotted in Fig. 4 as a function of the strain rate for a large range of filter sizes. The maximum resolved (unfiltered) flame temperature is virtually constant up to $\epsilon=10000 \mathrm{~s}^{-1}$ and decreases with larger strain rates, until extinction. For a constant strain rate, the maximum temperature of filtered flames decreases as filter size is increased. The maximum temperature decreases as well for a constant filter when the strain rate increases because the unfiltered flame thickness reduces and consequently the ratio $\delta_{\bar{Z}} / \delta_{Z}$ increases. The filtered flame structure thus dependent on both the filter size and the strain rate. This property is observed even for moderate strain rates, where the flame is nearly at equilibrium $\left(\epsilon<10000 \mathrm{~s}^{-1}\right)$, as shown in Fig. 4. Again, these conclusions are retrieved by the analytical analysis detailed in appendix, where a Gaussian shape spatial distribution is assumed to describe the resolved temperature profile. The analytical solutions of $\bar{T}_{\max }$ assuming finite rate and infinitely fast chemistry are added to Fig. 4. The influence of the filter size on the maximal filtered temperature is qualitatively recovered by the analytical expressions, even under infinitely fast chemistry assumption.

\subsection{Mapping the flame structure of filtered flames}

Filtered flame structures, well characterized by the maximal temperature and thickness, are thus dependent on both the filter size and the strain rate. A set of controlling variables needed to monitor this filtered flame structure is now identified. For that purpose we introduce the progress variable defined as [15]: $Y_{c}=Y_{\mathrm{CO}_{2}}+Y_{\mathrm{CO}}$. 

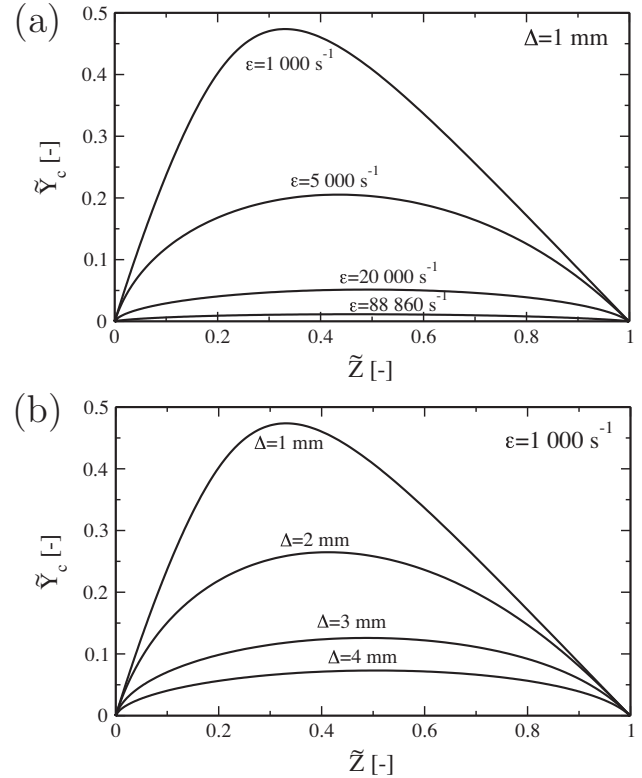

Fig. 5. Progress variable mass fraction $\tilde{Y}_{c}$ in terms of the mixture fraction $\tilde{Z}$. (a) The filter is set to $\Delta=1 \mathrm{~mm}$ and the strain rate is varied. (b) The strain rate is set to $\epsilon=1000 \mathrm{~s}^{-1}$ and filter size $\Delta$ is varied.

Figure 5(a) and (b) plots the filtered flame chemical trajectories in the plane $\left(\tilde{Y}_{c}, \tilde{Z}\right)$ for different strain rates and filter sizes, respectively. The chemical trajectories do not overlap in the phase subspace, meaning that both the set of coordinates $\left(\tilde{Z}, \Delta, \tilde{Y}_{c}\right)$ and $(\tilde{Z}, \Delta, \epsilon)$ are adapted to capture the diffusion filtered flame structure as in [16,6]. As $\tilde{Y}_{c}$ can be estimated from the solution of a balance equation, the triplet $\left(\tilde{Z}, \Delta, \tilde{Y}_{c}\right)$ is here preferred.

\section{F-TACLES for non-premixed combustion}

\subsection{Filtered balance equations: modeling issues in tabulated chemistry LES}

For LES, under unity Lewis assumption, the reactive flow governing equations read [11]:

$$
\begin{aligned}
& \frac{\partial \bar{\rho}}{\partial t}+\nabla \cdot(\bar{\rho} \tilde{\mathbf{u}})=0 \\
& \frac{\partial \bar{\rho} \tilde{\varphi}}{\partial t}+\nabla \cdot(\bar{\rho} \tilde{\mathbf{u}} \tilde{\varphi})=\nabla \cdot\left(\rho_{0} D_{0} \nabla \tilde{\varphi}\right)+\alpha_{\varphi}+\Omega_{\varphi}+\overline{\dot{\omega}}_{\varphi} \\
& \frac{\partial \bar{\rho} \tilde{\mathbf{u}}}{\partial t}+\nabla \cdot(\bar{\rho} \tilde{\mathbf{u}} \tilde{\mathbf{u}})=-\nabla \bar{p}+\nabla \cdot \bar{\tau}+\Omega_{u}
\end{aligned}
$$

$$
\begin{aligned}
\frac{\partial \bar{\rho} \tilde{E}}{\partial t}+\nabla \cdot(\bar{\rho} \tilde{\mathbf{u}} \tilde{E})= & -\nabla \bar{p} \tilde{\mathbf{u}}+\nabla \cdot \overline{(\tau \mathbf{u})} \\
& +\nabla \cdot\left(\rho_{0} D_{0} \nabla \tilde{h}_{s}\right)+\alpha_{h_{s}}+\Omega_{H}+\bar{\omega}_{E}
\end{aligned}
$$

where $\varphi$ denotes a chemical database coordinate (here $Z$ and $\tilde{Y}_{c}$ ), u the velocity vector, $D$ the molecular diffusivity, $\rho_{0}$ and $D_{0}$ reference values for the density and the molecular diffusion coefficient, $E=H-p / \rho$, with $H$ the total non-chemical enthalpy, $h_{s}$ the sensible enthalpy, $\dot{\omega}_{\varphi}$ and $\dot{\omega}_{E}$ the chemical database and energy source terms. It will be shown in Section 4.1 that the unresolved laminar convective contribution for the momentum equation $\Omega_{u}=-\nabla \cdot(\overline{\rho \mathbf{u u}}-\bar{\rho} \tilde{\mathbf{u}} \tilde{\mathbf{u}})$ can actually be neglected as in [11] and thus will not be detailed here. The functions $\alpha_{\varphi}, \Omega_{\varphi}$ and $\overline{\dot{\omega}}_{\varphi}$ are the unresolved diffusion, convective and chemical contributions:

$$
\begin{aligned}
& \alpha_{\varphi}=\nabla \cdot\left(\overline{\rho D \nabla \varphi}-\rho_{0} D_{0} \nabla \tilde{\varphi}\right) \\
& \Omega_{\varphi}=-\nabla \cdot(\overline{\rho \mathbf{u} \varphi}-\bar{\rho} \tilde{\mathbf{u}} \tilde{\varphi})
\end{aligned}
$$

An adequate closure of Eqs. (9) and (10) is now proposed to accurately monitor the filtered flame thicknesses and filtered maximal temperatures behavior presented previously. Issues relative to the numerical resolution of the filtered flame structure are also discussed.

\subsection{F-TACLES closure for filtered counter-flow} flames with resolved mixture fraction surface wrinkling

In the present approach, the flame structure is assumed similar to a planar filtered counter-flow flame computed for a given value of strain rate $\epsilon$. The wrinkling of $\tilde{Z}$ is fully resolved at the subgrid scale. Within the F-TACLES formalism [11], the unclosed terms are estimated from the explicit filtering of 1-D laminar non-premixed counter-flow flames and stored in a look-up table. In the following, symbols noted with * refer to 1-D counter-flow solutions.

The velocity vector $\mathbf{u}$ is decomposed in two contributions: the velocity of the flame front $\mathbf{w}$ (localized on a given iso- $Z$ and constant over the flame) and the local velocity in the flame front $\mathbf{v}$, so that $\mathbf{u}=\mathbf{v}+\mathbf{w}$. The coordinates in the local basis $(\mathbf{n}, \mathbf{t})$ of the flame front are $\left(x_{n}, x_{t}\right)$. The vector normal to the flame front is given by $\mathbf{n}=\nabla \tilde{Z} /|\nabla \tilde{Z}| . \mathbf{t}$ is perpendicular to $\mathbf{n}$ so that $\mathbf{v}=v_{n} \mathbf{n}+v_{t} \mathbf{t}$.

\subsubsection{Unresolved diffusion terms}

Under the assumption that $\nabla Z$ and $\nabla \varphi$ are aligned, the unresolved diffusion terms may be directly expressed from a filtered 1-D laminar counter-flow flame solution:

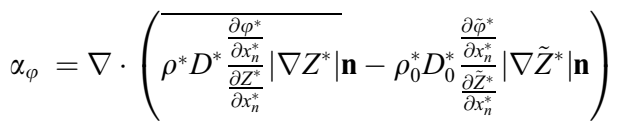

$$
\begin{aligned}
& =\nabla \cdot\left(\overline{\rho^{*} D^{*} \frac{\partial \varphi^{*}}{\partial x_{n}^{*}} \operatorname{sign}\left(\frac{\partial Z^{*}}{\partial x_{n}^{*}}\right)} \mathbf{n}\right) \\
& -\nabla \cdot\left(\rho_{0}{ }^{*} D_{0}^{*} \frac{\partial \tilde{\varphi}^{*}}{\partial x_{n}^{*}} \operatorname{sign}\left(\frac{\partial \tilde{Z}^{*}}{\partial x_{n}^{*}}\right) \mathbf{n}\right)
\end{aligned}
$$




\subsubsection{Unresolved convective terms}

The unresolved contribution for the scalar $\varphi$ can be recast as:

$$
\begin{aligned}
\Omega_{\varphi} & =-\nabla \cdot(\overline{\rho(\mathbf{v}+\mathbf{w}) \varphi}-\bar{\rho}(\tilde{\mathbf{v}}+\tilde{\mathbf{w}}) \tilde{\varphi}) \\
& =-\nabla \cdot(\overline{\rho \mathbf{v} \varphi}-\bar{\rho} \tilde{\mathbf{v}} \tilde{\varphi})
\end{aligned}
$$

as $\mathbf{w}$ is constant over the flame front. In local coordinates $\left(x_{n}, x_{t}\right)$ :

$$
\begin{aligned}
\Omega_{\varphi} & =\frac{\partial}{\partial x_{n}}\left(\overline{\rho v_{n} \varphi}-\bar{\rho} \tilde{v}_{n} \tilde{\varphi}\right)+\frac{\partial}{\partial x_{t}}\left(\overline{\rho v_{t} \varphi}-\bar{\rho} \tilde{v}_{t} \tilde{\varphi}\right) \\
& =\frac{\partial}{\partial x_{n}}\left(\overline{\rho^{*} u_{n}^{*} \varphi^{*}}-\overline{\rho^{*}} \tilde{u}_{n}^{*} \tilde{\varphi}^{*}\right)+\frac{\partial}{\partial x_{t}}\left(\overline{\rho^{*} u_{t}^{*} \varphi^{*}}-\overline{\rho^{*}} \tilde{u}_{t}^{*} \tilde{\varphi}^{*}\right)
\end{aligned}
$$

Variables in Eq. (16) are computed from a 1D counter flow flame (Section 2.2), where $\varphi^{*}=\varphi^{*}\left(x_{n}\right), u_{n}^{*}=u_{n}^{*}\left(x_{n}\right)$ and $u_{t}^{*}=U^{*}\left(x_{n}\right) \in x_{t}$, leading to:

$$
\begin{aligned}
\Omega_{\varphi}= & -\frac{\partial}{\partial x_{n}}\left(\overline{\rho^{*} u_{n}^{*} \varphi^{*}}-\overline{\rho^{*}} \tilde{u}_{n}^{*} \tilde{\varphi}^{*}\right) \\
& \left.-\epsilon \overline{\rho^{*} \varphi^{*} U\left(x_{n}\right)^{*}}-\overline{\rho^{*}} \tilde{\varphi}^{*} U\left(\tilde{x}_{n}\right)^{*}\right)
\end{aligned}
$$

\section{Numerical applications}

The methodology derived in Section 3 is applied here on counter-flow diffusion flame configurations. It is first tested in a 1-D counter-flow solver in Section 4.1 under Low Mach number flow assumptions. Finally, filtered 2-D counterflow flames are simulated in a multi-dimensional compressible solver in Section 4.2.

A filtered chemical look-up table is generated from a collection of 1-D counterflow flame solutions, computed with detailed chemistry, using the GRI 3.0 mechanism and unitary Lewis assumption. The database is built for strain rates ranging from $\epsilon=250 \mathrm{~s}^{-1}$ to $30000 \mathrm{~s}^{-1}$ by step of $250 \mathrm{~s}^{-1}$ and for $\Delta$ ranging from $0.5 \mathrm{~mm}$ to $6 \mathrm{~mm}$ in steps of $0.5 \mathrm{~mm}$. For both 1-D and 2-D cases, the thermochemical quantities are computed as in [17].

Validation simulations of pure methane/pure oxygen diffusion flames are performed under the same conditions than Section 2.1.

\subsection{1-D counterflow flames}

The F-TACLES model developed in Section 3 is implemented in a 1-D counterflow flame solver. The domain is composed of 250 nodes, with regular spacing and the extends of the domain are $x_{n, \text { min }}=-4 \mathrm{~mm}$ and $x_{n, \max }=4 \mathrm{~mm}$. Filtered flamelets solutions obtained with the F-TACLES model are compared with reference solutions obtained from the explicit filtering of resolved solutions.

\subsubsection{Influence of the closure on momentum}

Figure 6 shows temperature and $\tilde{Z}$ profiles for the unfiltered field $(--)$, the reference filtered solution - and the solution predicted by $\mathrm{F}$ TACLES (घ) for a strain rate of $\epsilon=5000 \mathrm{~s}^{-1}$. The filtered solution is accurately recovered using the proposed model. To assess the sensitivity of the solution to the unresolved convective term $\Omega_{u}$ in the momentum balance equation, the same computation was performed imposing $\Omega_{u}=0$. Results presented in Fig. $6(\bullet)$ indicate that the assumption $\Omega_{u}=0$ does not implies significant error. Accounting or not for $\Omega_{u}$ affects the heat release by less than $1 \%$. As for premixed flame LES [11], $\Omega_{u}=0$ is imposed in the following computations.

\subsubsection{Variable strain rate and filter size}

The model is now tested for a large range of strain rates and filter sizes. The filter size is first varied in Fig. 7, for a constant strain rate $\epsilon=5000 \mathrm{~s}^{-1}$. Results are in good agreement with the reference filtered solution, both for $\tilde{Z}$ and $\tilde{T}$. Indeed, the filtered flame thickness and the filtered temperature profiles are well captured by the model, for all filter size values. Results where the strain rate is varied from $\epsilon=5000 \mathrm{~s}^{-1}$ to $30000 \mathrm{~s}^{-1}$ with $\Delta=2 \mathrm{~mm}$ are given in Fig. 8 . Again, results are in good agreement with the reference profiles and for large set of strain rates the flame thickness is properly controlled. It is worth to mention that as the unfiltered flame thickness is reduced with the strain rate, the filtered temperature show large profiles variations depending on $\epsilon$, as mentioned in Section 2.2.
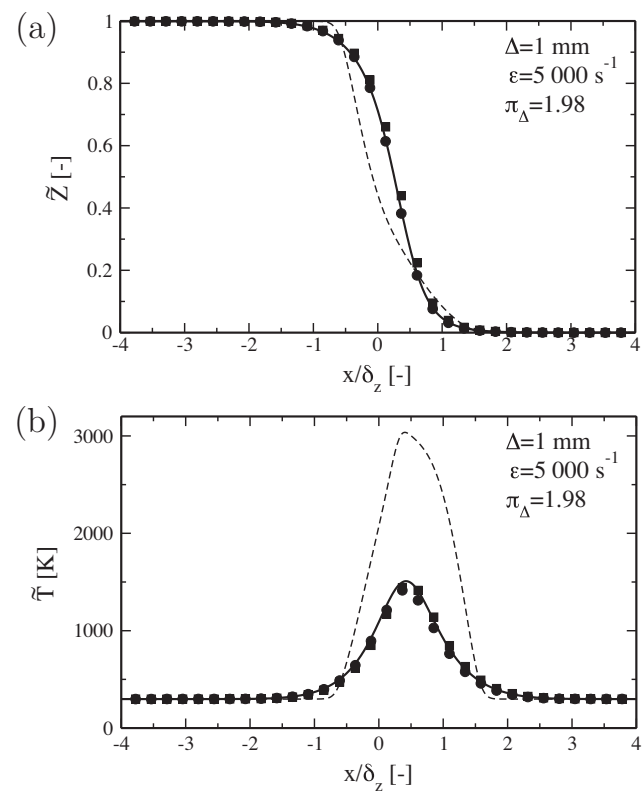

Fig. 6. Influence of $\Omega_{u}$. Numerical solution of $\tilde{Z}$ (a) and $\tilde{T}$ (b). - - Resolved solution - a priori filtered solution - a posteriori solution including $\Omega_{u}$ - a posteriori solution with $\Omega_{u}=0 . \pi_{\Delta}=\Delta / \delta_{z}$. 

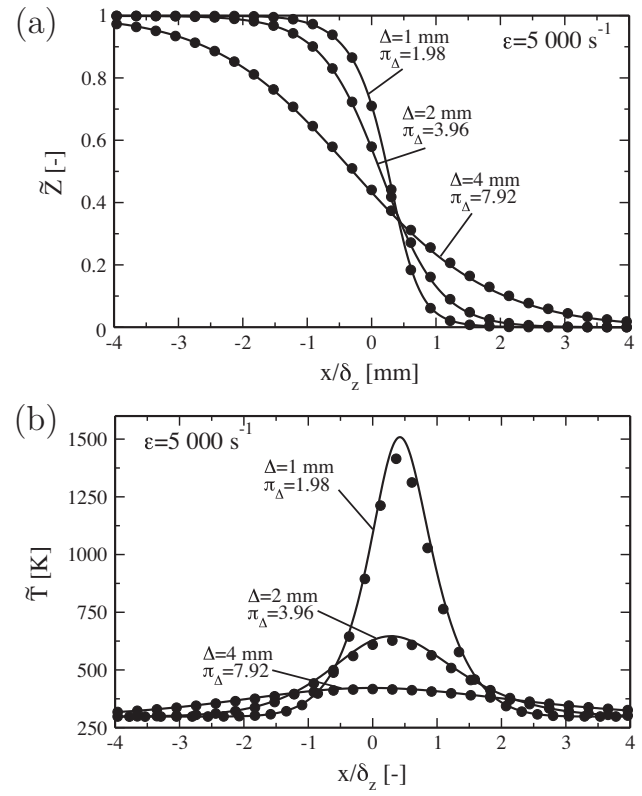

Fig. 7. Influence of the filter size. Numerical solution of $\tilde{Z}$ (a) and $\tilde{T}$ (b) for $\epsilon=5000 \mathrm{~s}^{-1}$. - a priori filtered solutions $\bullet$ a posteriori solutions. $\pi_{\Delta}=\Delta / \delta_{z}$.
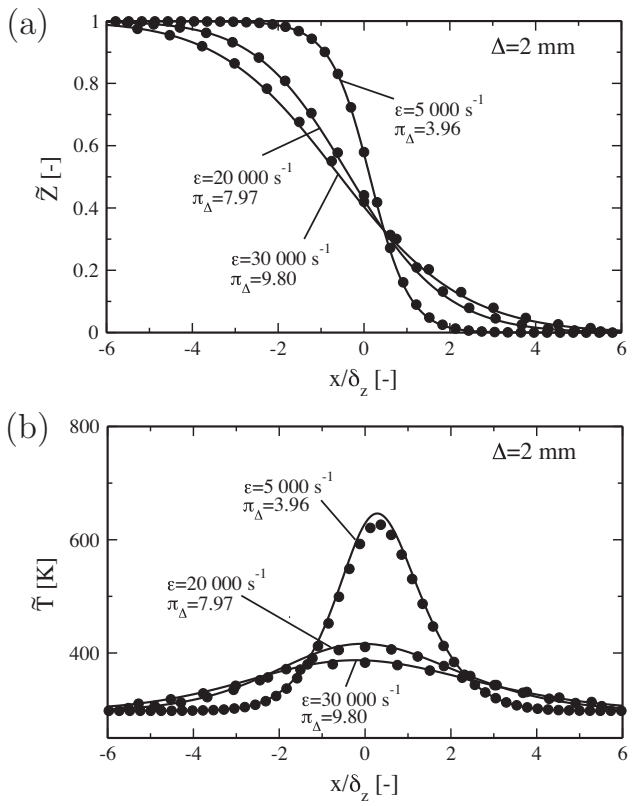

Fig. 8. Influence of the strain rate. Numerical solution of $\tilde{Z}$ (a) and $\tilde{T}$ (b) for $\Delta=2 \mathrm{~mm}$. - a priori filtered solutions $\bullet$ a posteriori solutions. $\pi_{\Delta}=\Delta / \delta_{z}$.

\subsection{2-D counterflow flames}

\subsubsection{Computational setup}

The model is implemented in the YWC compressible flow solver [18]. The conservation equations are solved using a fourth order finite difference scheme with a fourth order Runge-Kutta time integration. A 2-D counter-flow flame at a strain rate $\epsilon=7500 \mathrm{~s}^{-1}$ is computed. The mesh is composed of $640 \times 320$ nodes, with a spacing of $\Delta x_{n}=1.25 \times 10^{-5} \mathrm{~m}$ in the $x_{\mathrm{n}}$-direction and $\Delta x_{t}=2.5 \times 10^{-5} \mathrm{~m}$ in the $x_{\mathrm{t}}$-direction. The domain and inlet boundary conditions are detailed in Fig. 9. Outlets are imposed at $x=x_{\min }$ and $x=x_{\max }$ with a pressure of $p=101325 \mathrm{~Pa}$ using a 3-D NSCBC characteristic approach [19]. The filter size is chosen as $\Delta=2 \mathrm{~mm}$.

\subsubsection{Comparison with reference filtered solutions}

Computed flow streamlines are shown in Fig. 9. The mixture fraction $\tilde{Z}$ and temperature $\tilde{T}$ profiles extracted at $x_{t}=0 \mathrm{~mm}$ (cut 1 on Fig. 9) and $x_{t}=2 \mathrm{~mm}$ (cut 2 on Fig. 9) are plotted in Fig. 10. They both are in very good agreement with the expected profiles, obtained from a priori filtering of a resolved counter-flow flame solution. It is also demonstrated that the model behaves well on 2-D flows, as the profiles on cut 2 match accurately the reference solution.

\subsubsection{Influence of the grid resolution}

It is proposed to study the influence of the grid size on the quality of the computed solution. The mesh is progressively coarsened by successively dividing by two the grid size in both $x_{n}$ and $x_{t}$-directions. Figure 11 shows temperature $\tilde{T}$ profiles extracted at $x_{t}=2 \mathrm{~mm}$. The finer grid solution is compared with 3 coarsening levels corresponding to ratio between the filtered flame thickness and the grid spacing $\delta_{\bar{z}} / \Delta_{x}=15.6,7.8$ and 3.9 (this corresponds to ratio between the filter size and the grid spacing $\Delta / \Delta_{x}=20,10$ and 5). Good agreement is observed even with a grid resolution with only 4 cells in the flame front. This

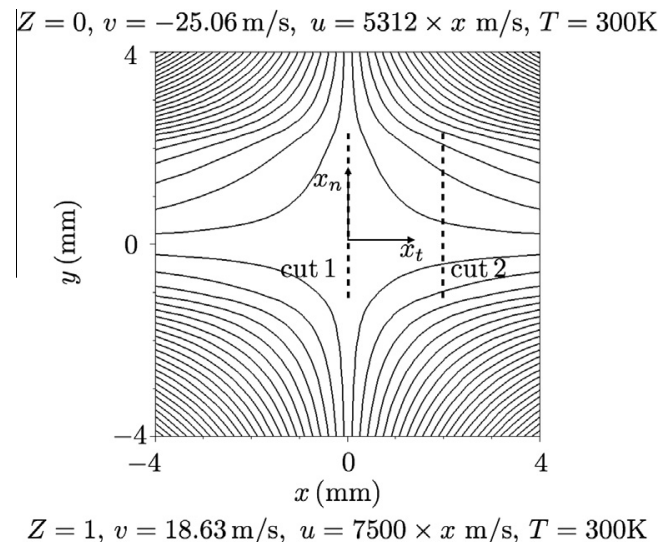

Fig. 9. 2-D counter-flow flames computational domain, where the stream lines are plotted. 

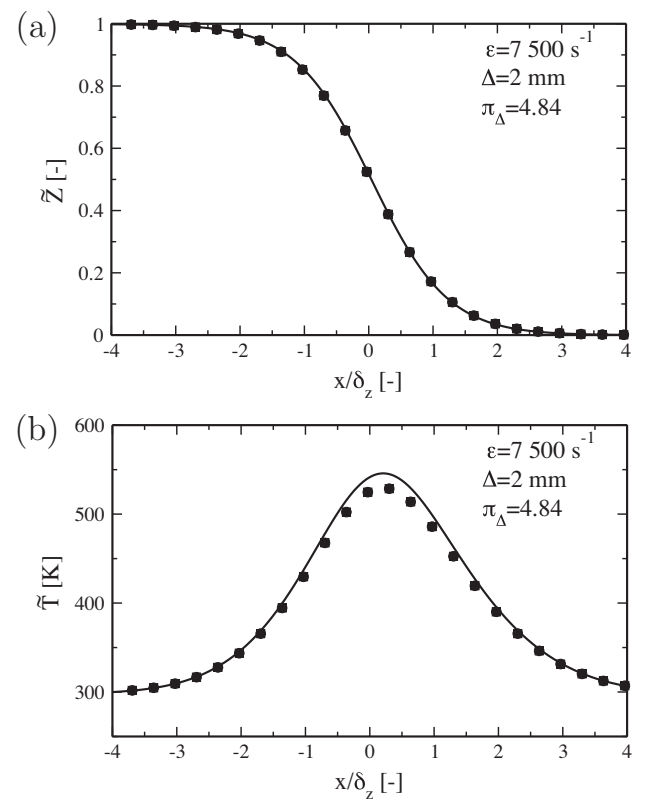

Fig. 10. Results from the 2-D compressible solver showing (a) $\tilde{Z}$ and (b) $\tilde{T}$ for $\Delta=2 \mathrm{~mm}$ and $\epsilon=7500 \mathrm{~s}^{-1}$. - a priori filtered solutions $\bullet$ a posteriori solutions at $x_{t}=0 \mathrm{~mm}$ - a posteriori solutions at $x_{t}=2 \mathrm{~mm} . \pi_{\Delta}=\Delta / \delta_{z}$.

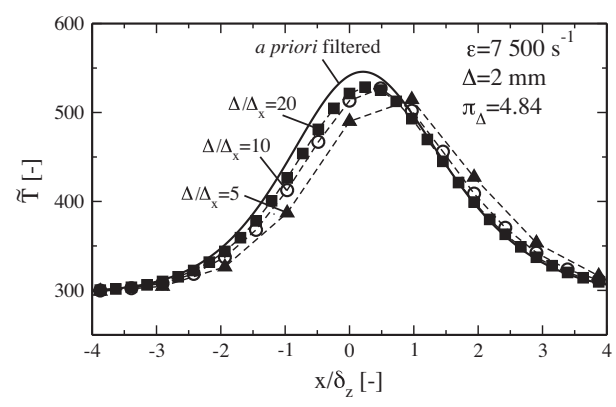

Fig. 11. Influence of the grid resolution Results from the 2-D compressible solver showing $\tilde{T}$ at $x_{t}=2 \mathrm{~mm}$. $-a$ priori filtered solution $\Delta / \Delta_{x}=20, \circ \Delta / \Delta_{x}=10$, $\Delta / \Delta_{x}=5 . \pi_{\Delta}=\Delta / \delta_{z}$

last grid resolution corresponds to commonly used LES grid resolution.

\section{Conclusions}

An extension of the F-TACLES model has been developed for non-premixed combustion. The unresolved laminar fluxes are modeled using a filtered chemical look-up table. The model is validated on 1-D and 2-D filtered laminar counterflow flames configurations. It is shown that the filtered flame thickness is monitored by three
$\tilde{Y}_{\tilde{Y}}$ controlling variables: the filtered progress variable $\tilde{Y}_{c}$, the filtered mixture fraction $\tilde{Z}$ and the filter width $\Delta$. It has been shown that the filter size governs the minimal thickness of the filtered thermal layer. The filter size $\Delta$ is set to ensure a sufficient resolution of the flame front. The model developments have been restricted to situations where no SGS flame wrinkling occurs. Future work will focus on accounting for interactions between flame and turbulence at the subgrid scale level.

\section{Acknowledgment}

This work is supported by SAFRAN Snecma Space Engines Division, the prime contractor of the Ariane launcher cryogenic propulsion system and CNES.

\section{Appendix A. Analytical modeling of filtered max- imum temperature and flame thickness}

The mixture fraction is modeled by $Z(x)=\frac{1}{2}\left(1+\operatorname{erf}\left(\frac{x}{\delta_{z}(\epsilon) / \sqrt{\pi}}\right)\right)$ where $\delta_{Z}(\epsilon)$, the flame thickness, is function of the strain rate $\epsilon$. Assuming a gaussian filtering operator of size $\Delta$, the filtered mixture fraction $\bar{Z}$ reads:

$\bar{Z}(x)=\frac{1}{2}\left(1+\operatorname{erf}\left(\frac{x}{\sqrt{\delta_{Z}^{2} / \pi+\Delta^{2} / 6}}\right)\right)$

The temperature is now modeled as $T(x)=$ $\left(T_{\max }-T_{\min }\right) \exp \left(-\frac{6 x^{2}}{\Delta_{0}^{2}}\right)+T_{\min }$, where $T_{\max }$ and $T_{\min }$ are maximum and minimum flame temperature, respectively and $\Delta_{0}^{2}=3 /(2 \ln (2)) \delta_{T}(\epsilon)^{2}$ with $\delta_{T}(\epsilon)$ the full width half maximum of temperature. The filtered temperature then reads:

$$
\begin{aligned}
\bar{T}(x)= & \left(T_{\max }-T_{\min }\right)\left(\frac{\Delta_{0}^{2}}{\Delta_{0}^{2}+\Delta^{2}}\right)^{1 / 2} \\
& \times \exp \left(-\frac{6 x^{2}}{\Delta_{0}^{2}+\Delta^{2}}\right)+T_{\min }
\end{aligned}
$$

Filtered flame thickness and maximum temperature are deduced from Eqs. (18) and (19):

$$
\bar{T}_{\text {max }}=\left(T_{\max }-T_{\min }\right)\left(\frac{1}{1+\frac{2 \ln (2)}{3} \frac{\Delta^{2}}{\delta_{T}^{2}}}\right)^{1 / 2}+T_{\min }
$$

$\delta_{\bar{Z}}=\sqrt{\delta_{Z}^{2}+\pi \frac{\Delta^{2}}{6}}$

Models for $\delta_{Z}(\epsilon)$ and $\delta_{T}(\epsilon)$ are required to close Eqs. (20) and (21). Two approaches based on infinitely fast and complex chemistry assumptions are 
tested, respectively. For infinitely fast chemistry, mixture fraction and thermal thicknesses are modeled by the relations $\delta_{T}=\delta_{T, 0} \sqrt{\epsilon^{0} / \epsilon}$ and $\delta_{z}=\delta_{z, 0} \sqrt{\epsilon^{0} / \epsilon}$, where $\delta_{T, 0}$ and $\delta_{z, 0}$ are reference unfiltered flame thicknesses for a given strain rate $\epsilon_{0}$. For complex chemistry assumption, the thicknesses $\delta_{Z}(\epsilon)$ and $\delta_{T}(\epsilon)$ are tabulated from detailed chemistry 1-D counter-flow laminar flame simulations.

\section{References}

[1] D. Haworth, Prog. Energy Combust. Sci. 36 (2011) $168-259$.

[2] P. Jenny, S. Pope, M. Muradoglu, D. Caughey, J. Comput. Phys. 166 (2001) 218-252.

[3] S. Navarro-Martinez, A. Kronenburg, Proc. Combust. Inst. 32 (2009) 1509-1516.

[4] A. Garmory, E. Mastorakos, Proc. Combust. Inst. 33 (2011) 1673-1680.

[5] H. Pitsch, H. Steiner, Phys. Fluids 12 (2000) 25412554.

[6] M. Ihme, H. Pitsch, Combust Flame 155 (2008) 7089.

[7] G. Singla, P. Scouflaire, C. Rolon, S. Candel, Proc. Combust. Inst. 30 (2005) 2921-2928.
[8] S. Candel, M. Juniper, G. Single, P. Scouflaire, C. Rolon, Combust. Sci. Technol. 178 (2006) 161-192.

[9] J. Oefelein, Proc. Combust. Inst. 30 (2005) 2929 2937.

[10] V. Yang, Proc. Combust. Inst. 28 (2000) 925-942.

[11] B. Fiorina, R. Vicquelin, P. Auzillon, N. Darabiha, O. Gicquel, D. Veynante, Combust. Flame 157 (2010) 465-475.

[12] P. Auzillon, O. Gicquel, N. Darabiha, D. Veynante, B. Fiorina, Combust. Flame 159 (8) (2012) 2704 2717.

[13] N. Darabiha, Combust. Sci. Technol. 86 (1992) 163 181.

[14] G.P. Smith, D.M. Golden, M. Frenklach, N.W. Moriarty, B. Eiteneer, M. Goldenberg, C. Thomas Bowman, R.K. Hanson, S. Song, W.C. Gardiner, Jr., V.V. Lissianski, Z. Qin, 2011. Available from: http://www.me.berkeley.edu/gri mech/.

[15] B. Fiorina, O. Gicquel, L. Vervisch, S. Carpentier, N. Darabiha, Combust. Flame 140 (2005) 147-160.

[16] C.D. Pierce, P. Moin, J. Fluid Mech. 504 (2004) 73 97.

[17] R. Vicquelin, B. Fiorina, S. Payet, N. Darabiha, O. Gicquel, Proc. Combust. Inst. 33 (2011) 1481-1488.

[18] A. Coussement, O. Gicquel, B. Fiorina, G. Degrez, N. Darabiha, J. Comput. Phys. 245 (2013) 259-280.

[19] C.S. Yoo, Y. Wang, A. Trouve, H.G. Im, Combust. Theory Model. 9 (2005) 617-646. 